\title{
Numerical Investigation of Fluid Flow and Heat Transfer Inside a 2D Enclosure with Three Hot Obstacles on the Ramp under the Influence of a Magnetic Field
}

\author{
Mohammad Mehdi Keshtkar \\ Department of Mechanical Engineering, Kerman Branch, \\ Islamic Azad University, Kerman, Iran
}

\author{
Mohammad Ghazanfari \\ Department of Mechanical Engineering, Kerman Branch, \\ Islamic Azad University, Kerman, Iran
}

\begin{abstract}
This paper focuses on solving the fluid flow and heat transfer equations inside a two-dimensional square enclosure containing three hot obstacles affected by gravity and magnetic force placed on a ramp using Boltzmann method (LBM) applying multiple relaxation times (MRT). Although, the Lattice Boltzmann with MRT is a complex technique, it is a relatively new, stable, fast and high-accurate one. The main objective of this research was to numerically model the fluid flow and ultimately obtaining the velocity field, flow and temperature contour lines inside a two-dimensional enclosure. The results and their comparisons for different types of heat transfer revealed that free or forced heat transfer has a considerable impact on the heat transfer and stream lines. This can be controlled by modifying the Richardson number. It is revealed that changing the intensity of the magnetic field (Hartman number) has an appreciable effect on the heat transfer.
\end{abstract}

Keywords-Lattice Boltzmann; multiple relaxation times; twodimensional enclosure; hot obstacles; ramp; Hartmann number

\section{INTRODUCTION}

In fluid flow analysis, three types of techniques may be applied: microscopic, mesoscopic and macroscopic ones. Microscopic techniques are not that effective for solving engineering problems. Macroscopic techniques are common in computational fluid dynamics but they often do not have analytical solutions. They are rather solved through numerical methods such as finite differences, finite volume and finite element in which the values of velocity, temperature and pressure are obtained in the different situations and times. Mesoscopic techniques lie between the previous two, involving a set of molecules as a particle rather than a single particle or molecule of fluid. These particles can move in the specified directions, so that their equations of motion can be statistically expressed by the distribution equations. The Lattice Boltzmann is one of the most important mesoscopic techniques in fluid flow analysis. In the recent years, due to its simplicity of programming, high capability and precise simulation, the
Lattice Boltzmann method has been adopted for the various problems including the single-phase flows, multi-phase flows, non-steady flows, flow in porous media and many other problems as a new approach to solving complex problems related to fluid dynamics.

The multiple relaxation time model was initially proposed in [1].This method is widely used in many important and complex engineering problems including multiphase flows [2], incompressible flows $[3,4]$ the passing flows through porous media [5]. In [6], authors examined the bounce back boundary condition in the Boltzmann method demonstrating that the bounce back is suitable for the non-slip boundary condition on stationary walls. This method was developed and problems were solved through a complex geometry [7, 8]. In [9], authors numerically studied the natural convection of an electric fluid in a rectangular enclosure in presence of a magnetic field. They found that convection effect is reduced in the presence of strong magnetic field. This was apparent as heat transfer by an increasing Hartmann number. In [10], authors examined the effect of uniform magnetic field on the natural convection in a nano-fluid. The results obtained confirmed the previous results and the only difference lied in the solution and the choice of three functions for flow, heat and magnetic field. In [11], authors conducted an experimental investigation on forced convective heat transfer of magnetic fluids in the presence of an alternating magnetic field. They concluded that the alternative magnetic fields are more influential than the uniform ones in the heat transfer. In [12], authors simulated a square enclosure containing a water-aluminum nano-fluid affected by a magnetic field using Lattice Boltzmann. The differences of their works were related to the temperature depending on the walls. They concluded that increasing the Rayleigh number results in the greater heat transfer and as the Hartman number increases the heat transfer decreases. In [13], authors examined a container with a removable lid influenced by a forced convection with the obstacles. In [14], authors examined a compartment with more than one obstacle 
influenced by a magnetic field. In [15], authors carried out an experimental study to investigate the convection heat transfer of $\mathrm{Fe} 304 /$ water nanofluid through a porous metal foam tube with uniform heat flux under the influence of constant magnetic field. In [16], authors focused on magnetite nanoparticles that were prepared via the solvothermal technique for further application as a nanofluid in heat transfer studies. The results showed that heat transfer properties of the examined magnetite nanofluids based on various concentrations of water and ethylene glycol were significantly enhanced under the influence of an alternating magnetic field.

The Hall and MHD effects on peristaltic flow of CarreauYasuda fluid in a convectively curved configuration was studied in [17]. Thermal radiation, Soret and Dufour effects were also accounted. Physical behaviors of velocity, temperature, concentration and streamlines were discussed with the help of graphical representation. In [18], authors investigated the hydro-thermal characteristics of ferrofluid (water and $\mathrm{Fe} 304$ ) in a rib-channel exposed to several non-uniform transverse magnetic fields generated by an electric current going through several wires located perpendicularly in the flow between ribs. In [19], authors investigated the heat transfer characteristics and the entropy generation rate of hybrid graphene-magnetite nanofluids under a forced laminar flow that were subjected to the permanent magnetic fields. The effect of magnetic field on the nanofluid flow inside a sinusoidal two-tube heat exchanger was investigated numerically in [20]. This study focuses on the influence of variable magnetic field in the heat transfer of heat exchanger while mixture is single phase. The effect of a permanent magnetic field on the heat transfer characteristics of hybrid graphene-magnetite nanofluids (hybrid nanofluid) under forced laminar flow was experimentally investigated in [21]. The experimental heat-transfer results indicated that the heat transfer enhancement of the hybrid nanofluid compared to the control fluid (distilled water) was negligible when no magnetic field was applied.

In [22], authors studied entropy generation due to natural convection in entrapped trapezoidal cavities filled with nanofluid under the influence of magnetic field was numerically. The convective heat transfer characteristics of Fe304/Water (3 vol\%) nanofluids were investigated under a parallel constant and uniform magnetic field in [23]. A rise in the heat transfer coefficient was observed when the temperature of the nanofluids was high, so compared with the effect of the chain-like structure, it was reported that the nanoparticle's Brownian motion had a major effect on the nanofluid heat transfer performance under a weak magnetic field. In [24], authors reported the magnetic field effect on natural convection heat transfer in a curved-shape enclosure. The obtained results were depicted in terms of streamlines and isotherms which showed the significant effects of Hartmann number on the fluid flow and temperature distribution inside the enclosure. Fe304water nanofluid flow in a cavity with constant heat flux was investigated using a control volume based finite element method (CVFEM) in [25]. Effects of Rayleigh and Hartmann numbers and volume fraction of Fe304 (nano-magnetite, an iron oxide) on flow and heat transfer characteristics were analyzed.

According to the authors' knowledge, the LBM-MRT method has not been used for the investigation of the effects of the magnetic field on a ramp with three hot obstacles in cavity with these boundary conditions. Also, the novelty of this study lies on the investigation of the effects of different Richardson numbers $(R i)$ (type of heat transfer mechanism) and the various Hartmann numbers $(\mathrm{Ha})$ at different angles $(\lambda)$ on hydrothermal behavior of mentioned problem.

\section{GEOMETRY AND PROBLEM STATMENT}

The problem includes a two-dimensional square enclosure with the length and width of $100 \mathrm{~mm}$ in which the left and right boundaries are isolated while the upper and lower boundaries have constant temperatures $\left(T_{c}=0\right)$. The enclosure is located on a ramp and inside the enclosure there are three hot obstacles by dimensions of $12.5 \times 12.5 \mathrm{~mm}$ with constant temperature $\left(T_{h}=1\right)$. The spaces between the obstacles have been filled by fluids with Prandtl of 0.7. All dimensions are shown in Figure 1. The fluid in the enclosure is assumed to be Newtonian, incompressible, and laminar. The momentum equation is written in the Boussinesq approximation. The thermophysical properties of fluid are constant. Analyzing this problem requires to solve momentum and energy equations by the LBM-MRT formulation.

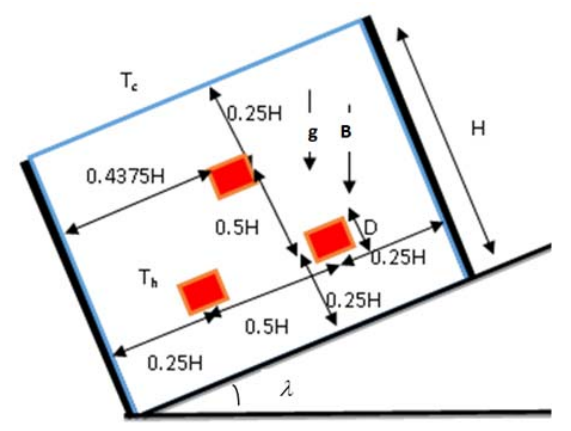

Fig. 1. Schematic view of the problem

\section{GOVERNING EQUATIONS}

The governing equations are mass, Navier-Stokes and the Energy equations and are written as follows:

$$
\begin{aligned}
& \nabla \cdot \mathrm{u}=0 \\
& \frac{\partial u}{\partial t}+u \cdot \nabla u=-\frac{1}{\rho_{0}}(\nabla P)+v \nabla^{2} u+F \\
& \frac{\partial T}{\partial t}+u . \nabla T=\nabla \cdot(\alpha \nabla T)
\end{aligned}
$$

The discrete form of kinetic equation for Lattice Boltzmann is formulated as follows: 


$$
\begin{aligned}
& \frac{f_{i}(x, t+\Delta t)-f_{i}(x, t)}{\Delta t}+c_{i} \cdot \frac{f_{i}(x+\Delta x, t+\Delta t)-f_{i}(x, t+\Delta t)}{\Delta x} \\
& =-\frac{1}{\tau}\left[f_{i}(x, t)-f_{i}^{e q}(x, t)\right]+F
\end{aligned}
$$

Where, $c_{i}$ is the velocity of the particle and $f_{i}$ is distribution function. Then, the distribution function represents the type of problem, which can vary depending on the problem. To solve the above equation, there are two steps, the first of which involves the impact obtained through the distribution function at equilibrium $\left(f_{i}{ }^{e q}\right)$. The distribution function at equilibrium state is obtained through the following equation:

$f_{i}^{e q}=\omega_{i} \rho\left(k_{1}+k_{2}\left(c_{i} \cdot u\right)+k_{3}\left(c_{i} \cdot u\right)+k_{4}(u \cdot u)\right)$

The $\mathrm{D}_{2} \mathrm{Q}_{9}$ method is used to solve the equation of momentum (Figure 2). Hence, there are 9 discrete velocities and 9 functions. The term $c$ represents the discrete velocity of particles, which are obtained according to the method of choice in (4).

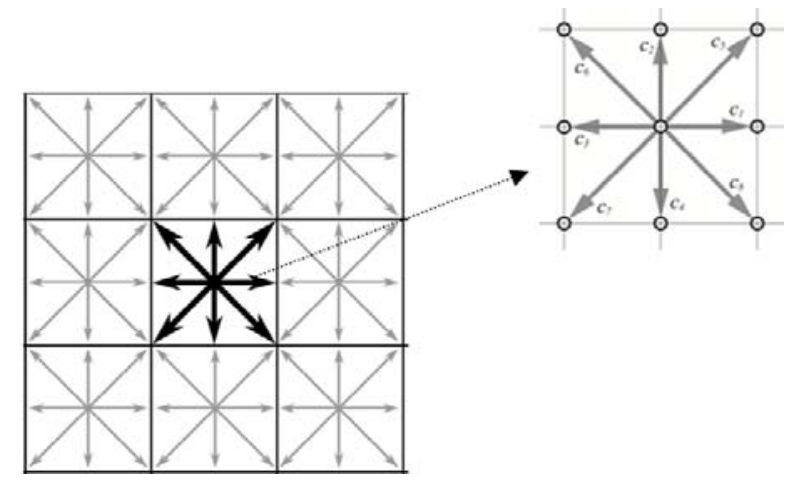

Fig. 2. D2Q9 layout

$$
\begin{aligned}
& c_{i}=\left(c_{i x}, c_{i y}\right), c_{0}=(0,0), c_{1}=(1,0), c_{2}=(0,1), c_{3}=(-1,0) \\
& c_{4}=(0,-1), c_{5}=(1,1), c_{6}=(-1,1), c_{7}=(-1,-1), c_{8}=(1,-1)
\end{aligned}
$$

In (3) weighting function $\omega$ is as follows:

$$
\omega=\left|\begin{array}{cc}
\frac{4}{9} & i=0 \\
\frac{1}{9} & i=1,2,3,4 \\
\frac{1}{36} & i=5,6,7,8
\end{array}\right|
$$

Where the constant terms in (3) are obtained by the following equations:

$$
k_{1}=1, k_{2}=\frac{1}{c_{c s}{ }^{2}}, k_{3}=\frac{1}{\left(2 c_{c s}{ }^{4}\right)}, k_{4}=-\frac{1}{2 c_{c s}{ }^{2}}
$$

The speed of sound $\left(c_{c s}\right)$ can be obtained from the following equation:

$$
c_{c s}=\frac{c}{\sqrt{3}}
$$

Fluid velocity vector is $\vec{u}$ and given as follows:

$$
\vec{u}=u_{i}+v_{j}
$$

According to (2), $F$ is the external force, which is the total force from gravity and magnetic force affecting the enclosure. Because of the slope of the surface, the external force should be placed on the main directions equal to the following [26]:

$$
\begin{aligned}
& F_{x}=\rho g \beta\left(T-T_{m}\right) \sin \lambda+\frac{H a^{2} \mu}{H^{2}}\left[v \sin \lambda \cos \lambda-u \sin ^{2} \lambda\right] \\
& F_{y}=\rho g \beta\left(T-T_{m}\right) \cos \lambda+\frac{H a^{2} \mu}{H^{2}}\left[u \sin \lambda \cos \lambda-v \cos ^{2} \lambda\right]
\end{aligned}
$$

The first term in the above equations represent the influence of gravity and the second term represents the effect of magnetic field analyzed based on the arrangement on the ramp in $\mathrm{x}$ and $\mathrm{y}$ directions. The Hartmann number represents $\mathrm{a}$ measure of the relative importance of MHD flow. Moreover, the various problems can be solved through MRT based on transfer matrix $(M)$. This matrix helps us arrive at a quicker solution by the moment vector space (m). Equations (10) and (11) represent the transfer matrix and the moment space matrix:

$$
\begin{aligned}
& M=\left(\rho, e, \varepsilon, j_{x}, q_{x}, j_{y}, q_{y}, p_{x x}, p_{y y}\right) \\
& m=\left(\rho, e, \varepsilon, j_{x}, q_{x}, j_{y}, q_{y}, p_{x x}, p_{y y}\right)^{T}
\end{aligned}
$$

The transfer matrix to solve the momentum equation is obtained as follows (in $\mathrm{D}_{2} \mathrm{Q}_{9}$ scheme) [27]:

$$
M=\left(\begin{array}{ccccccccc}
1 & 1 & 1 & 1 & 1 & 1 & 1 & 1 & 1 \\
-4 & -1 & -1 & -1 & -1 & 2 & 2 & 2 & 2 \\
4 & -2 & -2 & -2 & -2 & 1 & 1 & 1 & 1 \\
0 & 1 & 0 & -1 & 0 & 1 & -1 & -1 & 1 \\
0 & -2 & 0 & 2 & 0 & 1 & -1 & -1 & 1 \\
0 & 0 & 1 & 0 & -1 & 1 & 1 & -1 & -1 \\
0 & 0 & -2 & 0 & 2 & 1 & 1 & -1 & -1 \\
0 & 1 & -1 & 1 & -1 & 0 & 0 & 0 & 0 \\
0 & 0 & 0 & 0 & 0 & 1 & -1 & 1 & -1
\end{array}\right)
$$

With attention to the above equations, solving process begin from time step of $t=0$. After determining the initial 
values for vertical and horizontal speed and density, equilibrium distribution functions are obtained. The next stage is collision in which it is transferred to momentum space from the discrete space by the transfer matrix $(M)$ in the method of multiple-relaxation-time. The aim of this work is reduction of numerical errors. Collision stage in the Lattice-Boltzmann method with multiple-relaxation-time has the following steps:

- Conversion of the distribution functions $f$ from speed space to momentum space as $m=M f$.

- Collision in the momentum space, $m^{+}=m-\Lambda\left(m-m^{e q}\right)$.

- Conversion of distribution functions from the momentum space $\left(\mathrm{m}^{+}\right)$to speed space $\left(f^{+}\right)$as $f^{+}=M^{-1} m^{+}$.

After solving this stage and applying (2) (Streaming step), boundary conditions applied to the problem. Convergence condition (or a specified number of repetitions) is executed and the desired output is given. Furthermore, the transfer matrix to solve the energy equation is obtained as follow (in $\mathrm{D}_{2} \mathrm{Q}_{5}$ scheme) [26]:

$$
N=\left[\begin{array}{ccccc}
1 & 1 & 1 & 1 & 1 \\
0 & 1 & 0 & -1 & 0 \\
0 & 0 & 1 & 0 & -1 \\
-4 & 1 & 1 & 1 & 1 \\
0 & 1 & -1 & 1 & -1
\end{array}\right]
$$

These steps carried out for determination of the temperature filed, similarity. For the more information of exerting of the LBM-MRT readers can be refer to the references [27-28].

\section{BOUNDARY CONDITIONS}

If the problem boundary conditions imply that the enclosure walls are fixed and solid, the bounce back will be used on all the walls to solve the momentum equation. When solving the energy equation, we are faced with two boundary conditions: insulation boundary condition used for left and right insulation surfaces and constant temperature boundary condition used for the upper and lower surfaces.

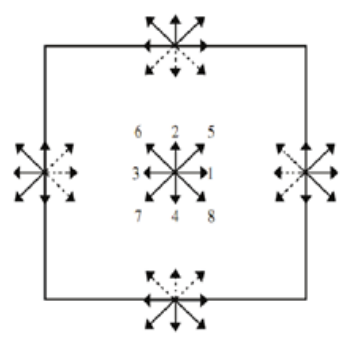

Fig. 3. Exerting Bounce Back in the momentum equation solving through D2Q9

Table I displays the boundary conditions based on the distribution functions when $\mathrm{D}_{2} \mathrm{Q}_{9}$ has been used. The formulation for insulated surfaces such as bounce back is done in the flow equation, whereas the boundary conditions for surfaces with constant temperature are formulated by the given relations in Table II.

TABLE I. BOUNDARY CONDITIONS (BOUNCE BACK) FOR MOMENTUM EQUATION [27]

\begin{tabular}{|c|c|c|c|}
\hline $\begin{array}{c}\text { Top } \\
\text { boundary }\end{array}$ & $\mathrm{f}(4,:, \mathrm{ly})=\mathrm{f}(2,:, \mathrm{ly})$ & $\mathrm{f}(7,:, \mathrm{ly})=\mathrm{f}(5,:, \mathrm{ly})$ & $\mathrm{f}(8,:, \mathrm{ly})=\mathrm{f}(6,:, \mathrm{ly})$ \\
\hline $\begin{array}{c}\text { Bottom } \\
\text { boundary }\end{array}$ & $\mathrm{f}(2,:, 1)=\mathrm{f}(4,:, 1)$ & $\mathrm{f}(6,:, 1)=\mathrm{f}(8,:, 1)$ & $\mathrm{f}(5,:, 1)=\mathrm{f}(7,:, 1)$ \\
\hline $\begin{array}{c}\text { Right } \\
\text { boundary }\end{array}$ & $\mathrm{f}(3, \mathrm{~lx},:)=\mathrm{f}(1,1 \mathrm{~lx},:)$ & $\mathrm{f}(6,1 \mathrm{~lx},:)=\mathrm{f}(8, \mathrm{~lx},:)$ & $\mathrm{f}(7,1 \mathrm{l},:)=\mathrm{f}(5,1 \mathrm{l},:)$ \\
\hline $\begin{array}{c}\text { Left } \\
\text { boundary }\end{array}$ & $\mathrm{f}(1,1,:)=\mathrm{f}(3,1,:)$ & $\mathrm{f}(8,1,:)=\mathrm{f}(6,1,:)$ & $\mathrm{f}(5,1,:)=\mathrm{f}(7,1,:)$ \\
\hline
\end{tabular}

TABLE II. BOUNDARY CONDITIONS FOR ENERGY EQUATIONS [26]

\begin{tabular}{|c|c|}
\hline $\begin{array}{c}\text { Top boundary } \\
\text { ( constant temperature ) }\end{array}$ & $\begin{array}{c}\mathrm{g}(4,1: \mathrm{lx}, \mathrm{ly})=0-\mathrm{g}(0,1: \mathrm{lx}, \mathrm{ly})-\mathrm{g}(1,1: \mathrm{lx}, \mathrm{ly})- \\
\mathrm{g}(2,1: \operatorname{lx}, \operatorname{ly})-\mathrm{g}(3,1: \mathrm{lx}, \mathrm{ly})\end{array}$ \\
\hline $\begin{array}{c}\text { Bottom boundary } \\
\text { ( constant temperature ) }\end{array}$ & $\begin{array}{c}\mathrm{g}(2,1: \operatorname{lx}, 1)=0-\mathrm{g}(0,1: \operatorname{lx}, 1)-\mathrm{g}(1,1: \operatorname{lx}, 1)- \\
\mathrm{g}(3,1: \operatorname{lx}, 1)-\mathrm{g}(4,1: \mathrm{lx}, 1)\end{array}$ \\
\hline $\begin{array}{l}\text { Right boundary } \\
\text { (Insulation ) }\end{array}$ & $\mathrm{g}(3, \mathrm{~lx},:)=\mathrm{g}(1, \mathrm{~lx},:)$ \\
\hline $\begin{array}{l}\text { Left boundary } \\
\text { ( Insulation ) }\end{array}$ & $\mathrm{g}(1,1,:)=\mathrm{g}(3,1,:)$ \\
\hline
\end{tabular}

For the obstacles with the constant temperature $\left(T_{h}=1\right)$, the boundary conditions should be formulated. To solve the momentum equations, the same bounce back is applied due to the solid surfaces. To solve the energy equation, the obstacle surfaces of the boundary condition for constant temperature are as follows [27]:

$$
g_{i}(x, t+\Delta t)=-g_{i}{ }^{c}(x, t)+2 \sqrt{3} \alpha T_{h}
$$

Where, $a$ and $T_{h}$ are the thermal diffusion coefficient and obstacle temperature, respectively.

\section{RESUlts}

\section{A. Independency of grid from meshing}

To show independency from the mesh, this problem was examined for four different mesh modes and the following results were obtained :

TABLE III. COMPARISON OF AVERAGE NUSSELT NUMBER IN DIFFERENT MESHING MODES

\begin{tabular}{|c|c|}
\hline Meshing & Average Nusselt number \\
\hline $71 \times 71$ & 3.6631 \\
\hline $101 \times 101$ & 3.6705 \\
\hline $121 \times 121$ & 3.6109 \\
\hline $151 \times 151$ & 3.6421 \\
\hline
\end{tabular}

One can conclude that the results are independent of the meshing. In this study, a $101 \times 101$ mesh was used to reduce the truncation error.

\section{B. Results and Discussion}

To ensure the correctness of the code, the results were compared with various references from the beginning of the process of solving, until sufficient confidence was built about 
the accuracy of the final results. The results obtained were compared with different references $[28,29]$ results and the entire accuracy of the code was assured.

TABLE IV. COMPARISON OF AVERAGE NUSSELT IN PRESENT WORK AND VARIOUS REFERENCES.

\begin{tabular}{|c|c|c|c|}
\hline $\begin{array}{c}\text { Grashof } \\
\text { number }\end{array}$ & $\begin{array}{c}\text { Khanafar's } \\
\text { study[28] }\end{array}$ & $\begin{array}{c}\text { Theoretical } \\
\text { research[29] }\end{array}$ & Present \\
\hline $10^{4}$ & 4.0430 & - & 4.1069 \\
\hline $10^{5}$ & - & 4.102 & 4.0068 \\
\hline
\end{tabular}

All results in the present study were based on forced $(R i \leq 0.1)$ and natural $(R i \geq 10)$ convection heat transfers in Reynolds number of 100 and $H a=0,10$ and 50 on the ramp at angles of $\lambda=0,30$ and 60 degrees as displayed in Figures 4 to 11. As shown, at $\lambda=0$ increasing the Hartmann number results in the more vortices formed between the lower obstacles. In contrast, there are no velocity contour lines at $H a=0$ (magnetic field is negligible) and there are zones with zero velocity. Transformation of velocity contour lines with the Hartmann number in a specified angle was evident for all states, especially for a Hartman number of 50 which was quite distinct. This indicates the high influence of the angulations on the enclosure at the high Hartmann number. When the Hartmann number is zero, there is little change in the overall shape of the velocity contour by changing the angle of the ramp especially for $\lambda=30$ and 60. However, $H a=10$ (weak magnetic field) led to more visible changes as high speed lines tended towards the center of the vortex enclosure near the upper obstacle. At $H a=50$, increasing the slope, results in stretching the angle of velocity contour lines which tend to form the third and fourth vortices at the upper right corner and bottom right corner of the enclosure. Moreover, increasing the slope of the ramp in forced convection was associated with the increase in velocity as shown in the pictures. At $\lambda=0$, increasing the Hartmann number causes the main vortex becomes gradually smaller while the lateral vortex around lower obstacles grows. By the angulations of the enclosure, at the first glance, the effect of the Hartmann number on the stream lines on the ramp is evident. In fact, the higher Hartmann number, the greater the angularity in the behavior of stream lines is; as if the center of the upper vortex is located on the left side of the higher obstacle as the vortex has been spun to the angle of the ramp anti-clockwise. This is more evident when the Hartman number is 50 . The flow field around the obstacles is created near the cold wall of the enclosure due to temperature difference between the hot and cold walls and then hits with adiabatic walls leading to change its direction toward the upper cold sidewall and then hits with the another cold wall. This recycles movement causes to produce the major vortices around the hot obstacles at the lower section of enclosure.

The comparison between Figures 4 and 6 visibly indicates that velocity contours in the free and forced convections are similar, and a high speed vortex around the upper obstacle is formed. In this case, it is noteworthy that as convection progresses towards free, these high speed lines are dragged towards the lower section of the enclosure. Also near the lower obstacles, a vortex is formed in the opposite direction of the previous vortex. It can be found in all two forms so that the Richardson number increases as velocity increases in stationary conditions. When the magnetic field effect is significant (i.e. $H a=50$ ), the Lorentz force due to magnetic field effect becomes higher than the buoyancy force due to the natural convection effect which leads to decrease the flow circulation strength and as a result the convection effect diminishes. As shown in Figure 7, in the free convection, the earlier facts applied and therefore the conditions do not change so drastically. However, a fundamental change occurs in the behavior of stream lines for $H a=0$ and $H a=10$. This change involves the formation of small vortices in the lower section of the enclosure between the two obstacles in where the vortices are eliminated with increased ramp angle to $\lambda=0$ degrees, turning into a vortex. In addition, the effect of angularity in this state is higher for $H a=0$ and $H a=10$ than the previous states. However, this angle is not even close to the minimum angle of the ramp and is far smaller than that. The formation of a small vortex at an angle of $\lambda=0$ at the top of the enclosure is due to the impact of the stream lines with the upper wall of the enclosure. In Figure 8, it can be seen that the arrangement of the isothermal temperature contour lines for forced convective heat transfer is the same in all cases. This reveals that changing the forced convective heat transfer into the Hartman number and angle of the ramp has very little effect on the behavior of the isothermal lines. Figure 9 shows clearly that the slope angle and the Hartman number have very little impact on changing trend of Nusselt number in forced convective heat transfer. Increasing the Hartman number influences the addition of the maximum Nusselt and increasing the ramp angle influences slightly the asymmetry of the forms. Another point is that the graphs are similar at $\lambda=0$ for all three modes. This reveals that changing the Hartmann number influences slightly the forced convective heat transfer. Figure 10 indicates that in the free convection heat transfer, changing the Hartmann number and the angle of the ramp has the higher effect than the forced heat transfer in such a way that in the certain circumstances the overall shape is totally transformed. As the enclosure angle increases the value of heat transfer increases. On the contrary, increasing the Hartman number for constant angle heat transfer will reduces. Figure 11 indicates that when the angle of the ramp is zero, the Nusselt number graphs have three extreme points on a regular basis and symmetrically shaped. With the angularity of the enclosure, however, the trend gradually alters and the maximum values of Nusselt number are closer to the right wall and the graph drifts apart from the symmetric mode. Another interesting point is the great similarity of Nusselt variations trend for $H a=0$ and $H a=10$. This reflects the fact that when the Hartman number becomes larger, the effect of changing the angle of the ramp on the Nusselt number is lower than when the Hartmann number is small. Also, this figure shows that, the values of Nusselt number decreases when the Hartmann number increases for $\lambda=0,30$ and increases for $\lambda=60$. This is due to the effect of magnetic field becomes very important. With increasing Hartmann number the flow circulation strength decreases and the temperature gradient reduces and for this reason the Nusselt number decreases. 
$\Sigma \varphi \alpha ́ \lambda \mu \alpha$ ! Н
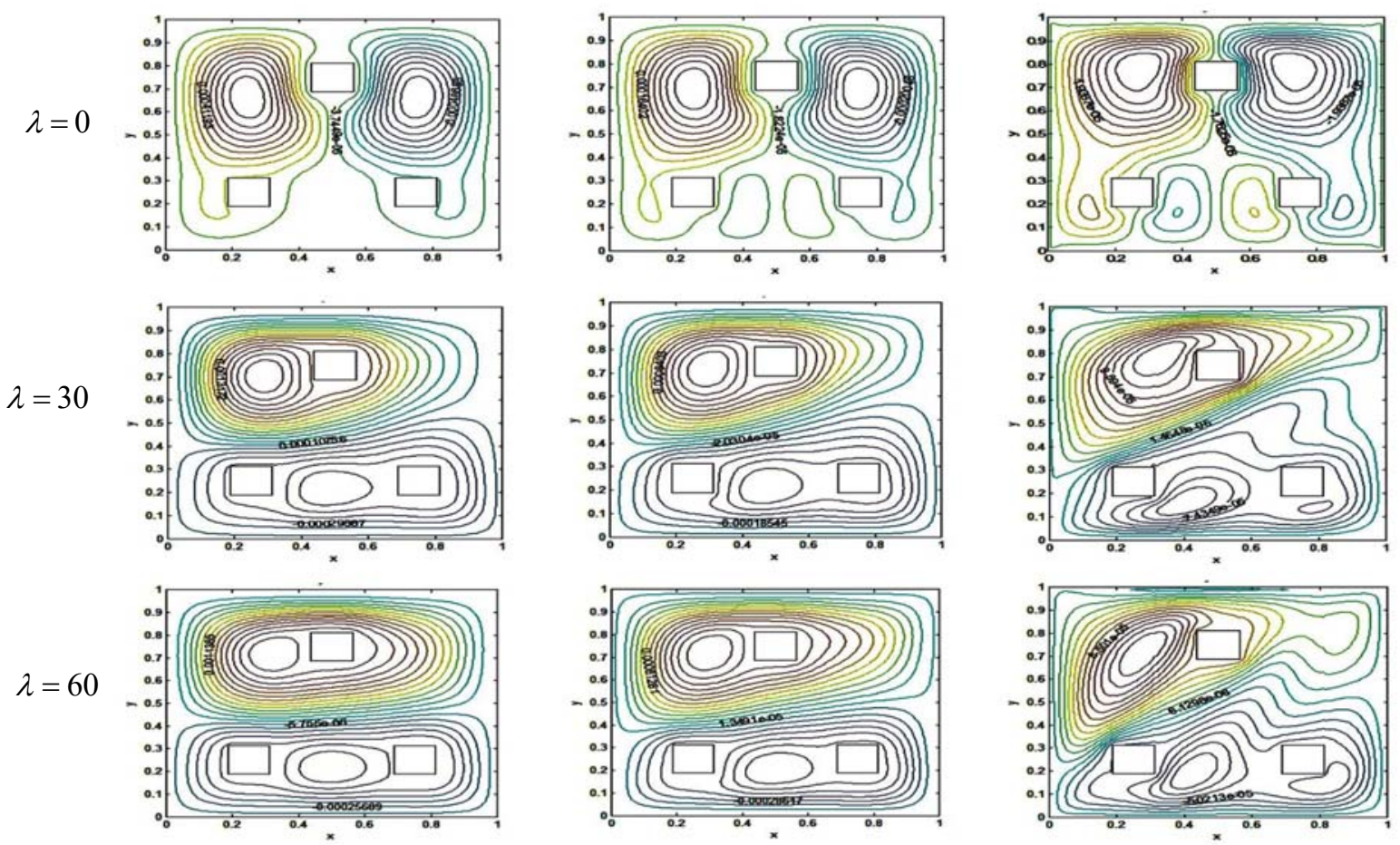

Fig. 4. Velocity contour in forced convection for different conditions.
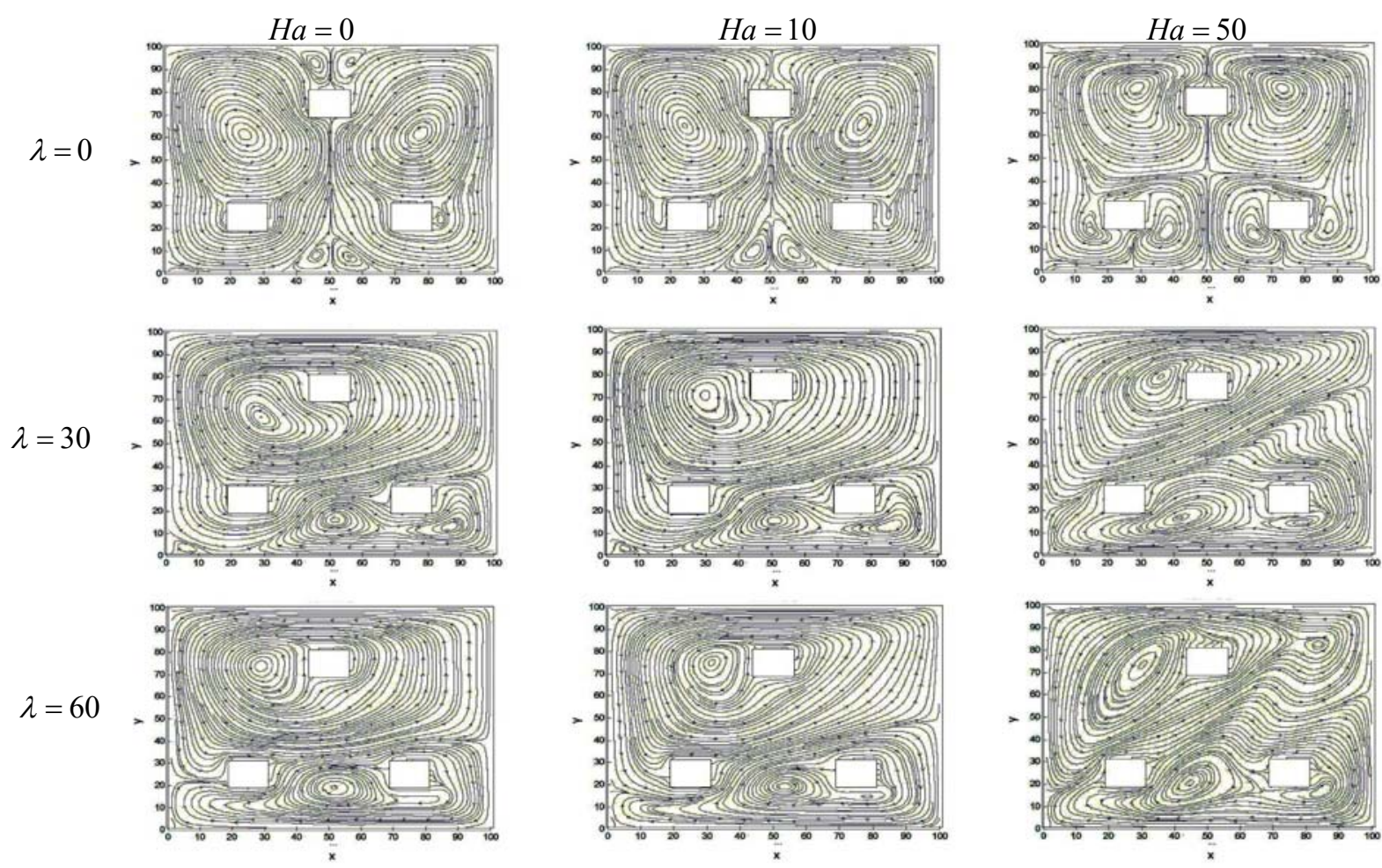

Fig. 5. Stream lines in forced convection for different conditions. 
$H a=0$
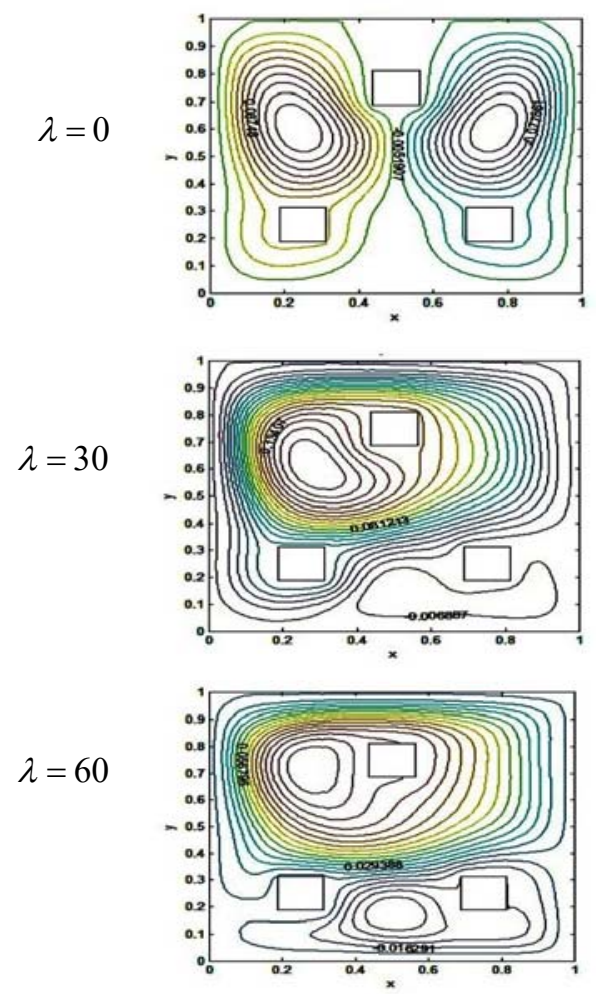

$H a=10$
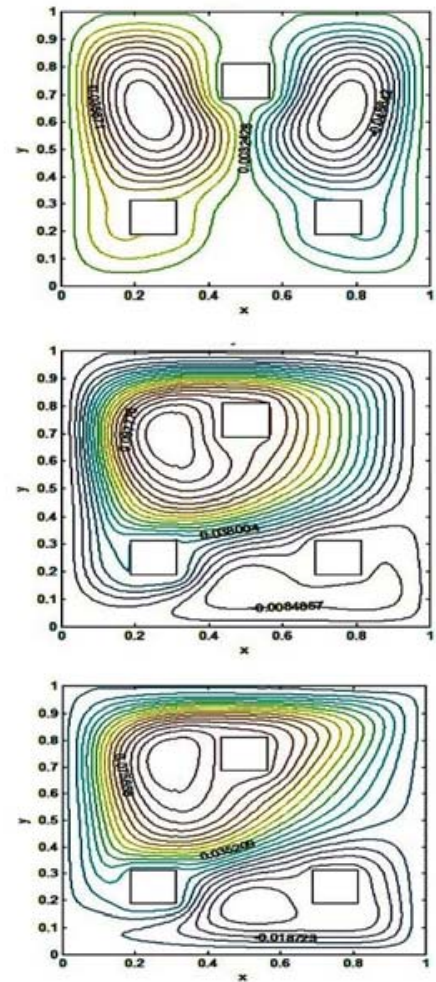

$H a=50$
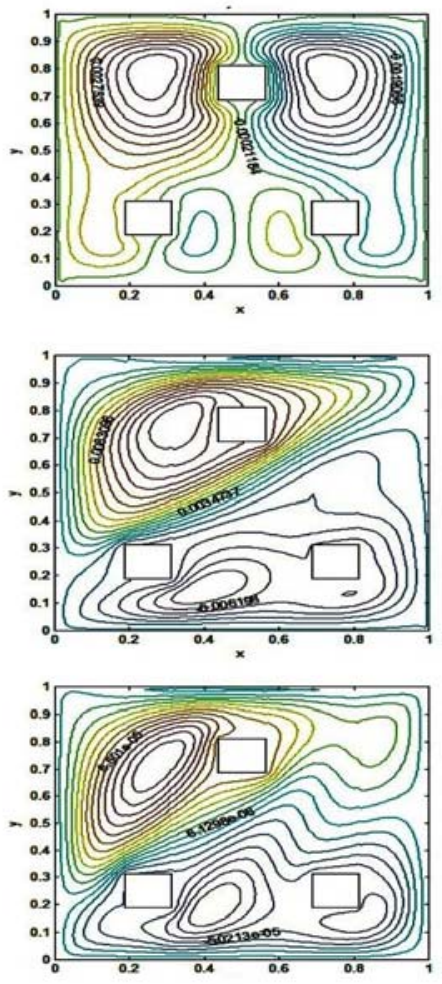

Fig. 6. Velocity contour speed in free convection for various conditions

$H a=0$
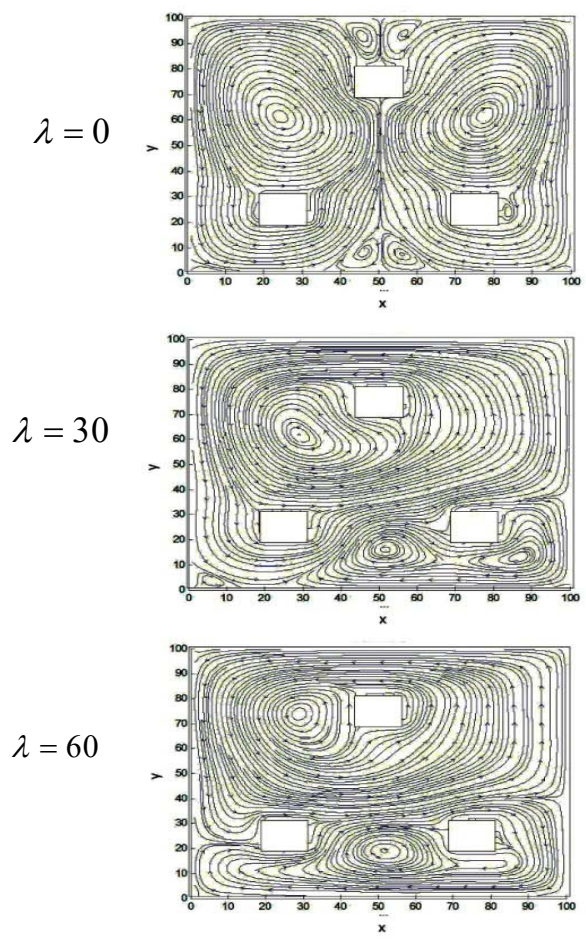

$$
H a=10
$$
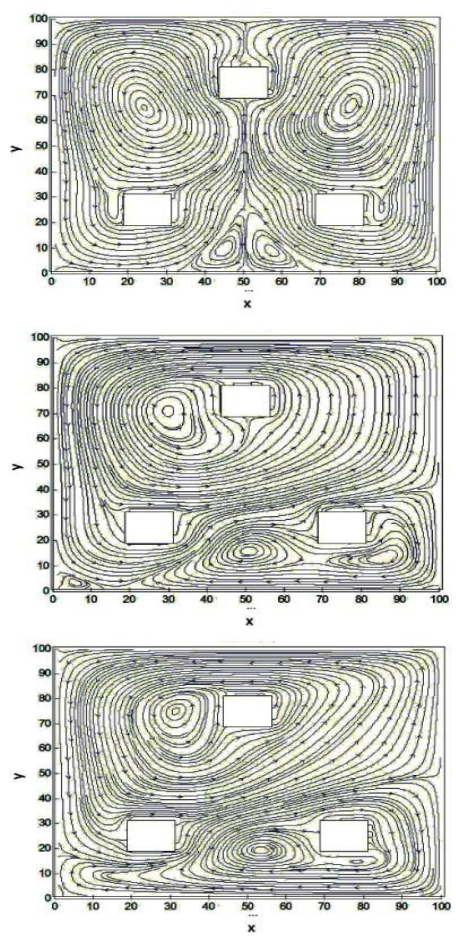

$H a=50$
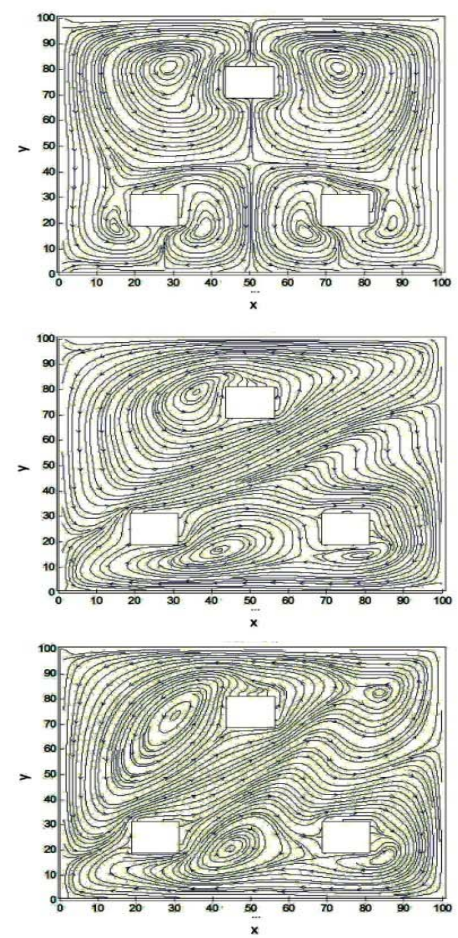

Fig. 7. Stream lines in free convection for different conditions. 

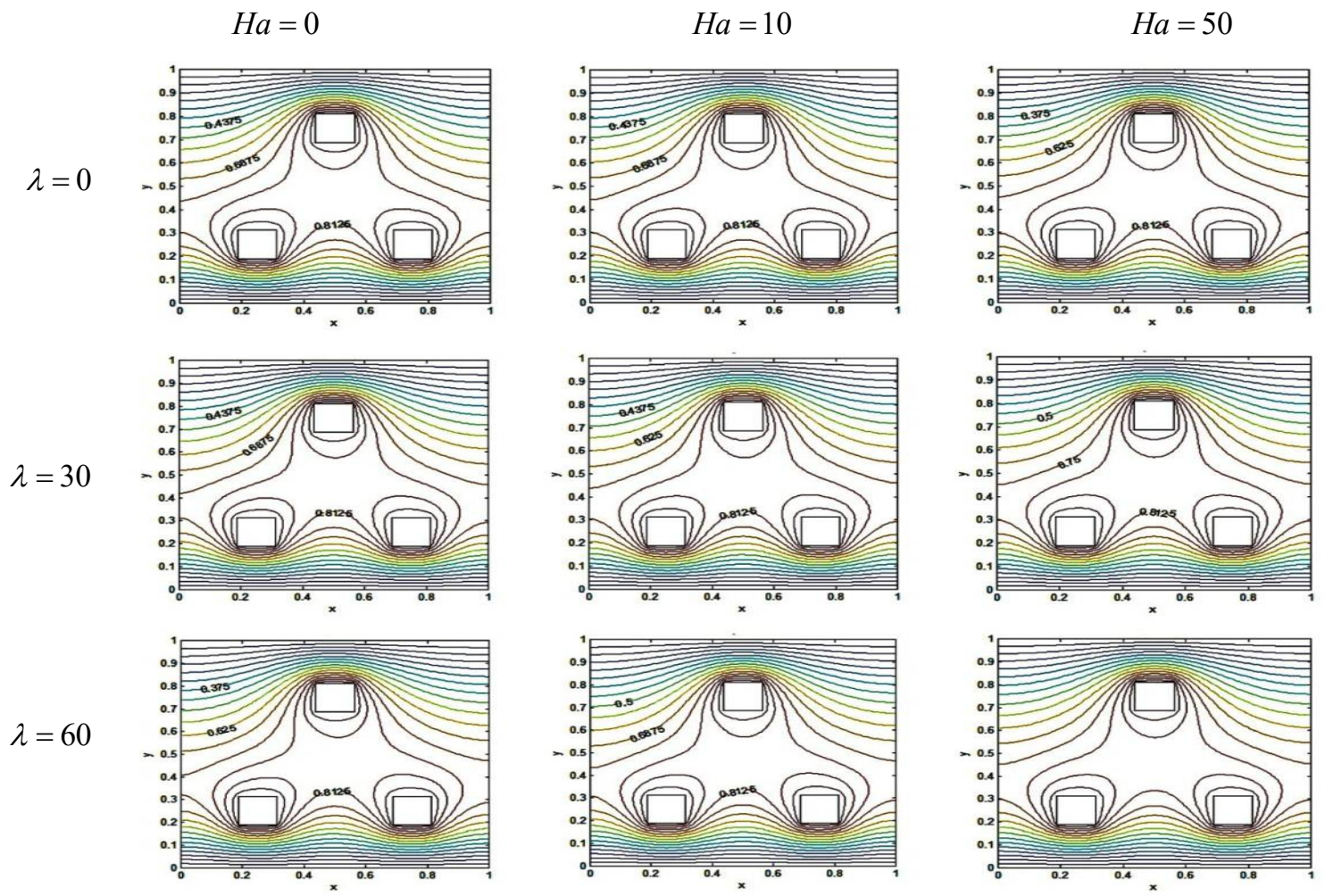

Fig. 8. Temperature contour in forced convection for different conditions.

$$
H a=0
$$
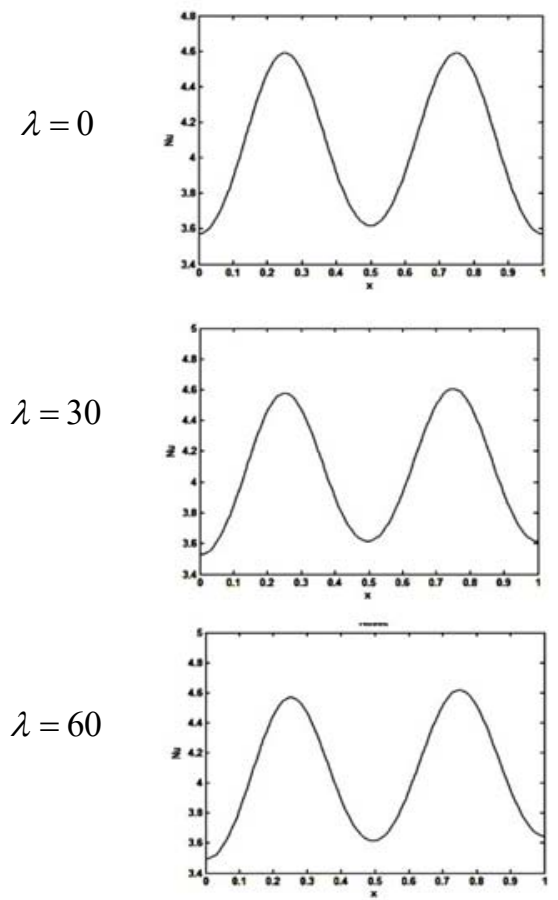

$H a=10$
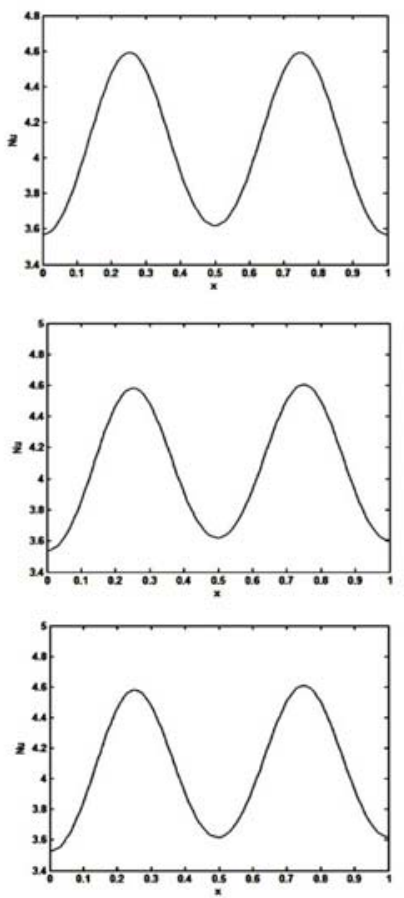

$H a=50$
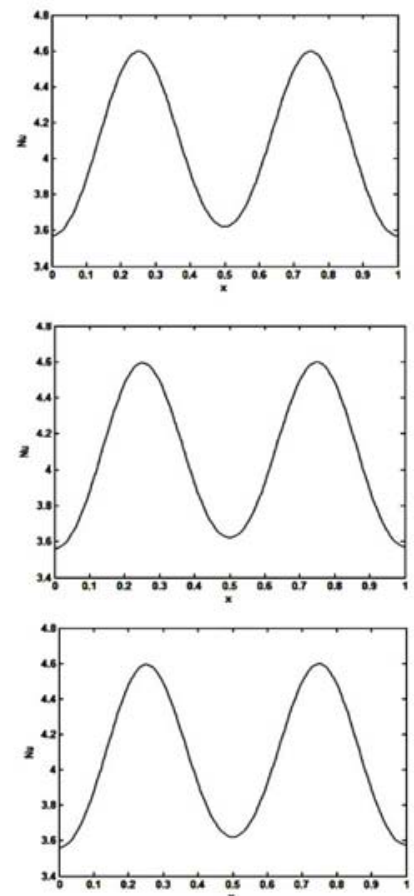

Fig. 9. Nusselt number variations in forced convection for different conditions. 
$H a=0$
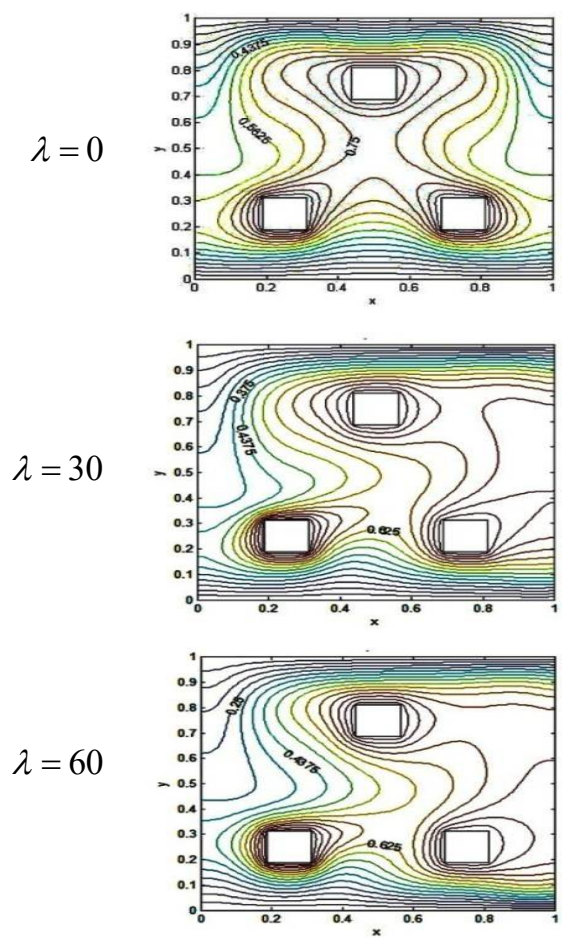

$H a=10$
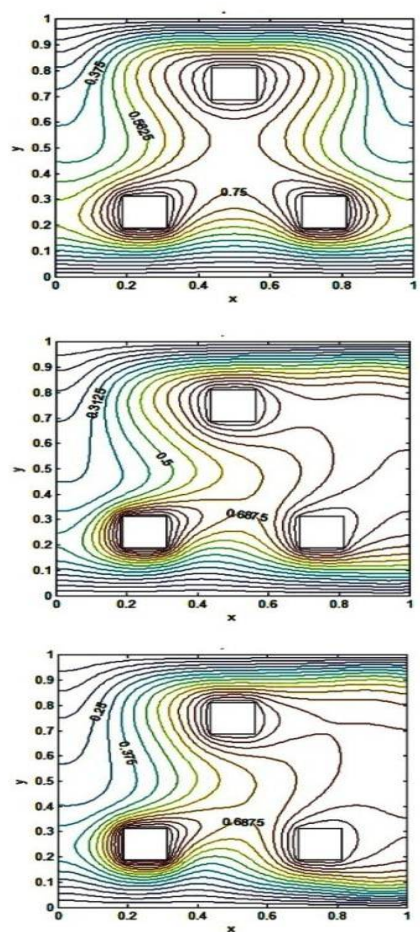

$H a=50$
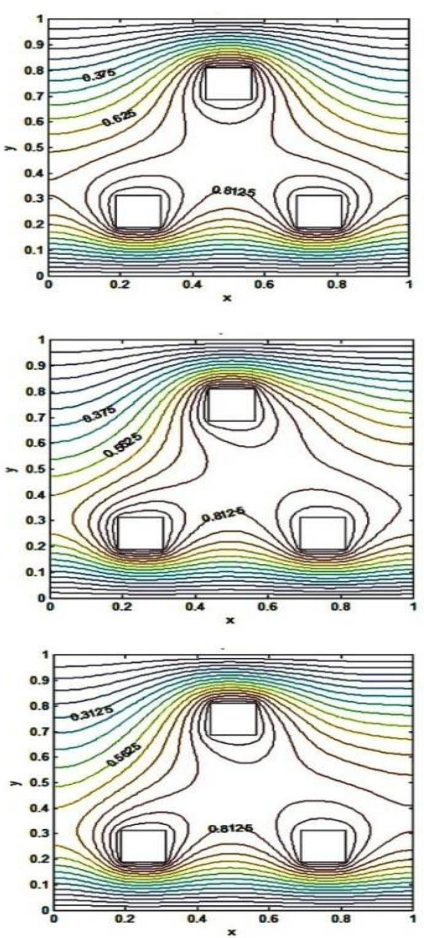

Fig. 10. Temperature contour in free convection for different conditions.

$H a=0$
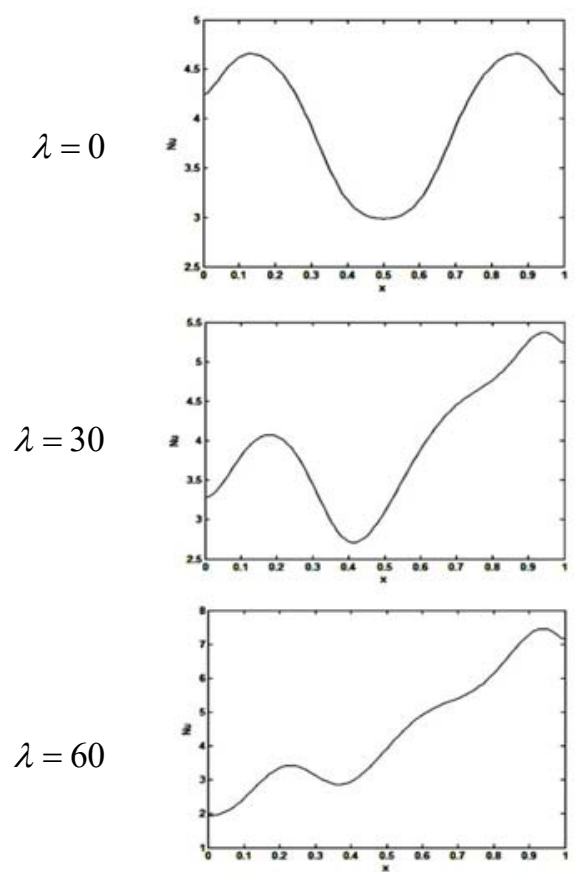

$H a=10$
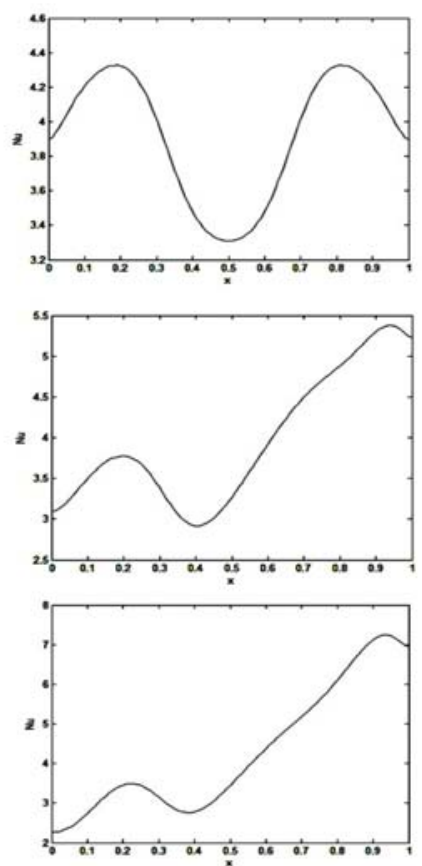

$H a=50$
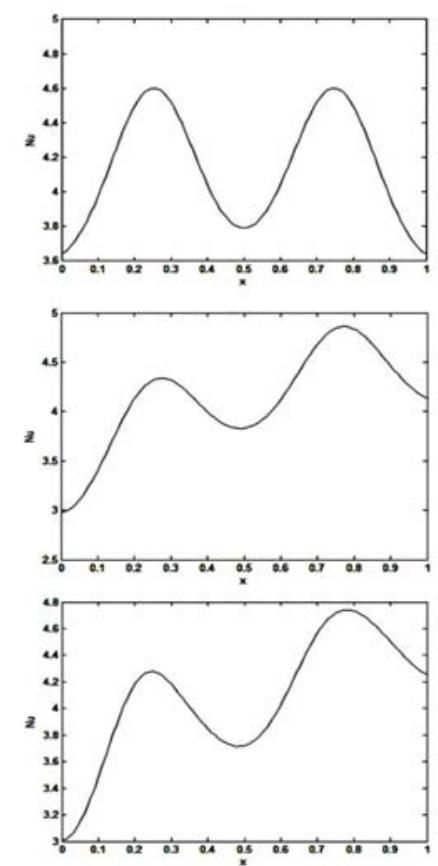

Fig. 11. Nusselt number variations in free convection for different conditions. 


\section{CONCLUSIONS}

The problem includes a two-dimensional square enclosure with a length and width of $100 \mathrm{~mm}$ in which the left and right boundaries are isolated while the upper and lower boundaries have constant temperatures. The enclosure is located on a ramp and inside the enclosure there are three hot obstacles with constant temperature. The results can be listed as follows:

- For a Hartman number of 50, the type of heat transfer (free or forced) does not have much impact on the shape of the velocity contour.

- The impact of the ramp angle on the stream lines for a Hartman number of 50 can be seen as much greater compared to lower Hartman numbers.

- In free convection heat transfer, the effect of changing the angle of the slope is much greater than that of the forced convection.

- In the forced convection heat transfer, a change in the angle of the ramp or a change in the Hartman number left little impact on the behavior of the temperature contour lines.

- In the free convection heat transfer, for a Hartman number of 0 and 10, as the angle of the slope increases, there will be greater heat transfer. For a Hartman number of 50, as the slope increases, there will be lower heat transfer.

- The rate of heat transfer in free convection is much higher than that in forced convection.

\section{NOMENCLATURE}

$B \quad$ Magnetic field

c Lattice velocity

g Gravitational acceleration, $\mathrm{m} \mathrm{s}^{-2}$

Gr Grashof number, $G r=g \beta\left(T_{s}-T_{m}\right) L^{3} / v^{2}$

$\mathrm{H}$ Enclosure length, $\mathrm{m}$

$\mathrm{Ha}$ Hartmann number, $H a=B H \sqrt{\sigma / \mu}$

$\mathrm{k}$ Thermal conductivity, $\mathrm{Wm}^{-1} \mathrm{~K}^{-1}$

$\mathrm{Nu}$ Nusselt number, $N u=h L / k$

Pr Prandtl number, $v / \alpha$

$\mathrm{Ra}$ Rayleigh number, $R a=G r$.Pr

Re Reynolds number, $V L / v$

$\mathrm{Ri}$ Richardson number, $R i=g \beta\left(T_{s}-T_{m}\right) L / V^{2}=G r / \mathrm{Re}^{2}$

$\mathrm{T}$ Dimensionless temperature, $\mathrm{K}$

$\mathrm{u}, \mathrm{V}$ Dimensionless velocities components in $\mathrm{x}$ and $\mathrm{y}$ direction, $\mathrm{m} \mathrm{s}^{-1}$

$V \quad$ Characteristic velocity, $\mathrm{m} \mathrm{s}^{-1}$

$\mathrm{x}, \mathrm{y}$ Dimensionless Cartesian coordinates, $\mathrm{m}$

Greek symbols $\alpha \quad$ Thermal diffusivity, $\mathrm{m}^{2} \mathrm{~s}$

$\beta \quad$ Thermal expansion coefficient, $\mathrm{K}^{-1}$

$\sigma \quad$ Electrical conductivity, $\mathrm{Sm}^{-1}$

$v \quad$ Kinematic viscosity, $\mathrm{m}^{2} \mathrm{~s}^{-1}$

\begin{tabular}{ll}
\multicolumn{2}{l}{ Subscripts } \\
c & Cold \\
f & Fluid \\
h & Hot \\
m & Mean \\
W & Wall
\end{tabular}

\section{REFERENCES}

[1] D. H. Rothman,S. Zaleski, "Lattice-gas Model of Phase Separation: Interfaces, Phase Transitions, and Multiphase Flow", Rev. Mod. Phys., Vol. 66, No. 4, pp. 1417-1479, 1994

[2] S. Chen, G. Doolen, "Lattice Boltzmann Method for Fluid Flows", Ann. Rev. Fluid Mech., Vol. 30, pp. 329-364, 1998

[3] S. Succi, The Lattice Boltzmann Equation for Fluid Dynamics and Beyond, Clarendon, Oxford, 2001

[4] G. Bella, S. Ubertini, M. Bertolino, "Computational Fluid Dynamics for Low and Moderate Reynolds Numbers through the Lattice Boltzmann Method", Int. J. Comp. Num. Analysis Applications, Vol. 3, No. 1, pp. 83-115, 2003

[5] G. Bella, M. Presti, S. Succi, "Mass Transfer Improvements in Catalytic Converter Channels:a Hybrid BGK-Finite Volume Numerical Simulation Method", Society Automotive Engineers, Paper, No. 972907 , 1997

[6] Q. Zah, X. He, "On Pressure and Velocity Flow Boundary Conditions and Bounceback for the Lattice Boltzmann BGK Model", Physics of Fluids, Vol. 9, pp. 1591-1598, 1997

[7] O. Filippova, D. Hänel, "Grid refinement for latticeBGK Models”, J. Comput. Phys. Vol. 147, pp. 219- 228, 1998

[8] T. Lee, C. L. Lin, "A Characteristic Galerkin Method for Discrete Boltzmann Equation”, J. Comput. Phys., Vol. 171, pp. 336-356, 2001

[9] N. Rudraiah, R. Barron, M. Venkatachalappa, C. Subbaraya, "Effect of magnetic field on free convection in rectangular enclosure", International Journal of Engineering Science, Vol. 33, No. 8, pp. 10751084,1995

[10] H. Nemati, M. Farhadi, K. Sedighi, H. Ashorynejad, E. Fattahi, "Magneticfield effects on natural convection flow ofnanofluid in rectangular cavity using the Lattice Boltzmann model", Scientia Iranica, Vol. 19, No. 2, pp. 303-310, 2012

[11] A. Ghofrani, M. Dibaei, A. Hakim Sima, M. Shafii, "Experimental Investigation on laminar forced convection heat transfer of ferro fluids under an alternating magnetic field", Experimental Thermal and Fluid Science, Vol. 49, pp. 193-200, 2013

[12] I. Mejri, A. Mahmoudi, M. A. Abbassi, A. Omri, "Lattice Boltzmann Simulation of MHD Natural Convection in Nanofluid-Filled EnclosureWith Non-Uniform Heating on Both SideWalls", International Journal of Mathematical, Computational, Natural and Physical Engineering, Vol. 8,No. 1, pp. 75-91, 2014

[13] M. Hemmat Esfe, M. Akbari, A. Karimipour, "Mixed Convection in a Lid-Driven Cavity with an Inside Hot Obstacle Filled by an Al2O3Water Nanofluid", J. Applied Mechanic and Thermal Physics, Vol. 56, No. 3, pp. 443-453, 2015

[14] T. Zhang, D. Che, "Double MRT thermal lattice Boltzmann simulation for MHD naturalconvection of nanofluids in an inclined cavity with four square heatsources", J. Heat and Mass Transfer, Vol. 94, pp. 87-100, 2016

[15] M. Amani, M. Ameri, A. Kasaeian, "Investigating the convection heat transfer of $\mathrm{Fe} 3 \mathrm{O} 4$ nanofluid in a porous metal foam tube under constant magnetic field", Experimental Thermal and Fluid Science, Vol. 82, pp. 439-449, 2016 
[16] E. Esmaeili, R. Ghazanfar Chaydareh, S. A. Rounaghi, "The influence of the alternating magnetic field on the convective heat transfer properties of Fe3O4-containing nanofluids through the Neel and Brownian mechanisms", Applied Thermal Engineering, Vol. 110, pp. 1212-1219, 2017

[17] T. Hayat, S. Farooq, A. Alsaedi, B. Ahmad, "Hall and radial magnetic fi eld effects on radiative peristaltic fl ow of Carreau - Yasuda fl uid in a channel with convective heat and mass transfer", Journal of Magnetism and Magnetic Materials, Vol. 412, pp. 207-216, 2016

[18] M. M. Larimi, A. Ghanaat, A. Ramiar, A. A. Ranjbar, "Forced convection heat transfer in a channel under the influence of various nonuniform transverse magnetic field arrangements", International Journal of Mechanical Sciences, Vol. 118, pp. 101-112, 2016

[19] M. Mehrali, E. Sadeghinezhad, A. R. Akhiani, S. Tahan Latibari, H. S. C. Metselaar, A. S. Kherbeet, M. Mehrali, "Heat transfer and entropy generation analysis of hybrid graphene/ $\mathrm{Fe} 3 \mathrm{O} 4$ ferro-nanofluid flow under the influence of a magnetic field", Powder Technology, Vol. 308, pp. 149-157, 2016

[20] S. V. Mousavi, M. Sheikholeslami, M. Gorji Bandpy, M. Barzegar Gerdroodbary, "The Influence of magnetic field on heat transfer of magnetic nanofluid in a sinusoidal double pipe heat exchanger", Chemical Engineering Research and Design, Vol. 113, pp. 112-124, 2016

[21] E. Sadeghinezhad,M. Mehrali, A. R. Akhiani, S. Tahan Latibari, A. Dolatshahi-Pirouz, H. S. C. Metselaar, M. Mehrali, "Experimental study on heat transfer augmentation of graphene based ferrofluids in presence of magnetic field", Applied Thermal Engineering, Vol. 114, pp. 415427,2017
[22] F. Selimefendigil, H. F. Oztop, N. Abu-Hamdeh, "Natural convection and entropy generation in nanofluid filled entrapped trapezoidal cavities under the influence of magnetic field", Entropy, Vol. 18, No. 2, pp. 122, 2016

[23] L. Sha, Y. Ju, H. Zhang, J. Wang, "Experimental investigation on the convective heat transfer of $\mathrm{Fe} 3 \mathrm{O} 4 /$ water nanofluids under constant magnetic field", Applied Thermal Engineering, Vol. 113, pp. 566-574, 2017

[24] M. Sheikholeslami, I. Hashim, S. Soleimani, "Numerical Investigation of the Effect of Magnetic Field on Natural Convection in a CurvedShape, Vol 34, pp. 56-64, 2013

[25] M. Sheikholeslami, K. Vajravelu, "Nanofluid flow and heat transfer in a cavity with variable magnetic field", Applied Mathematics and Computation, Vol. 298, pp. 272-282, 2017

[26] J. Wanga, D. Wanga, P. Lallemand, L. Luoc, "Lattice Boltzmann simulations of thermal convective flows in two dimensions", Computers and Mathematics with Application, Vol. 65, pp. 262-286, 2013

[27] C. Rettinger, Fluid flow simulations using the lattice Boltzmann method with multiple relaxation times, Bachelor Thesis, Friedrich-AlexanderUniversität Erlangen-Nürnberg, Germany, 2013

[28] K. Khanafer, K. Vafai, M. Lightstone, "Buoyancy-driven heat transfer enhancement in two-dimensional enclosure utilizing nanofluids", International Journal of Heat and Mass Transfer, Vol. 46, No. 19, pp. 162-186, 2003

[29] M. Nazari, S. Ramzani, "Natural Convection in a Square Cavity with a Heated Obstacle Using Lattice Boltzmann Method", J. Heat and Mass Transfer, Vol. 32, pp. 127-133, 2011 